\title{
Autonomic hyperreflexia associated with recurrent cardiac arrest: Case Report
}

\author{
SC Colachis, III $^{1}$ and DM Clinchot ${ }^{2}$ \\ ${ }^{1}$ Associate Professor and ${ }^{2}$ Assistant Professor, Department of Physical Medicine and Rehabilitation ${ }^{1}$, Director, SCI \\ Rehabilitation, The Ohio State University, College of Medicine, Columbus, Ohio, USA
}

\begin{abstract}
Autonomic hyperreflexia is a condition which may occur in individuals with spinal cord injuries above the splanchnic sympathetic outflow. Noxious stimuli can produce profound alterations in sympathetic pilomotor, sudomotor, and vasomotor activity, as well as disturbances in cardiac rhythm. A case of autonomic hyperreflexia in a patient with C6 tetraplegia with recurrent ventricular fibrillation and cardiac arrest illustrates the profound effects of massive paroxysmal sympathetic activity associated with this condition.
\end{abstract}

Keywords: autonomic hyperreflexia; spinal cord injury; ventricular fibrillation

\section{Introduction}

Autonomic hyperreflexia is a condition of paroxysmal reflex sympathetic activity which occurs in response to noxious stimuli in patients with spinal cord injuries above the major splanchnic sympathetic outflow. ${ }^{1-3}$ The heightened sympathetic activity during an episode of autonomic hyperreflexia accounts for several of the clinical features commonly observed including sudomotor and pilomotor phenomenon, ${ }^{1,4-6}$ vasomotor sequelae, ${ }^{1-4,7}$ and alterations in cardiac inotropic and chronotropic activity. $1,2,4,6,7$

Cardiac abnormalities described in association with episodes of autonomic hyperreflexia include bradycardia $^{1,4,6,7}$ and tachycardia, 2,6,7 premature atrial and ventricular contractions, ${ }^{1,8}$ atrial fibrillation ${ }^{9,10}$ and conduction block. ${ }^{8}$ Despite the occurrence of these arrhythmias associated with autonomic hyperreflexia, they are seldom recognized as a cause of cardiac arrest in individuals with traumatic spinal cord injury.

The following case of autonomic hyperreflexia with recurrent ventricular fibrillation and cardiac arrest illustrates the profound effects of massive paroxysmal sympathetic activity associated with this condition.

\section{Case report}

A 28 year old male with C6 motor and sensory complete tetraplegia (ASIA Impairment Scale 'A') from a motor vehicle accident in 1983 experienced an episode of cardiac arrest on 3 July 1994. He had been experiencing recurrent symptoms of autonomic hyperreflexia since the prior evening. His care attendant noted increased symptoms of autonomic hyperreflexia that morning during personal care activities manifested

Correspondence: Sam C Colachis, M.D. by excessive sweating and flushing. Past episodes of autonomic hyperreflexia were generally attributed to reflex voiding, position changes and the presence of pressure sores.

The attendant had completed the patient's morning bowel program, hygiene and dressing activities, and started to exit the apartment when he heard gasping. $\mathrm{He}$ returned to find him pulseless, apneic, and cyanotic. There was no prior evidence of airway difficulty. He called the emergency medical personal and began basic cardiopulmonary resuscitation measures. After electrocardiography was made available, the emergency medical team found the patient to be in ventricular fibrillation. He was successfully cardioverted to sinus rhythm, intubated, and emergently transferred to our acute medical center.

Initial evaluation in the emergency department revealed no obvious etiology (e.g. hypoxemia, hypovolemia, or metabolic disturbances) for the cardiac arrest. Toxicology studies were unremarkable. However, wide variability in blood pressure was recorded with pressures ranging from 108/66-170/76 $\mathrm{mmHg}$. Approximately $90 \mathrm{~min}$ after arrival, during close observation, his rhythm returned to ventricular fibrillation and he again had a cardiac arrest necessitating resuscitation efforts including cardioversion. He was successfully resuscitated and was transferred to the medical intensive care unit. A pulmonary angiogram was normal.

The patient's initial hospital course was complicated by wide variations in systolic and diastolic blood pressure which improved over the next few days, and an aspiration pneumonia which resolved with treatment. He slowly regained consciousness but was confused for several days. This was attributed to anoxic encephalopathy which subsequently resolved. An initial echocardiogram on 4 July 1994 revealed an 
ejection fraction of only $27 \%$ with apical akinesia. The etiology for this was felt to be cardiac shock. A repeat examination performed on 7 July 1994 showed an ejection fraction of $55 \%$. Cardiac catheterization on 8 July 1994 was normal with a 64\% ejection fraction.

On 11 July 1994, he underwent electrophysiological studies which revealed no inducible ventricular tachycardia, normal sinus node, and no accessory pathway. In view of the two prior documented episodes of ventricular fibrillation and cardiac arrest, the patient opted for an implantable internal cardiac defibrillator which was surgically placed on 13 July 1994. He was subsequently discharged home.

Our patient is currently enrolled in continuing college education. He has had no further episodes of cardiac arrest and the implanted defibrillator has not discharged.

\section{Discussion}

Autonomic hyperreflexia is associated with profound alterations in vasomotor, ${ }^{1-4,7}$ pilomotor $^{4}$ and sudomotor activity. ${ }^{1,4-6}$ Heightened sympathetic activity associated with hypertensive crisis during an episode have resulted in seizures, ${ }^{3,4,6}$ retinal $^{4}$ and intracranial hemorrhages, ${ }^{3}$ cerebral vascular accidents, ${ }^{3,8}$ coma $^{3,6}$ and death. ${ }^{3,6}$ Resolution of this autonomic phenomenon occurs with removal of the noxious stimulus precipitating the event.

Cardiac abnormalities observed during an episode of autonomic hyperreflexia include inotropic and chronotropic changes. Palpitations, chest pain, ${ }^{1}$ dyspnea, ${ }^{6,11}$ congestive heart failure ${ }^{4}$ and pulmonary edema $^{11}$ have all been reported. Tachycardia ${ }^{2,6}$ and reflex bradycardia ${ }^{1,2,4,6}$ are most commonly observed in this setting, and are attributed to heightened noradrenergic sympathetic activity and reflex vagal responses, respectively. Electrocardiographic abnormalities observed during an attack of autonomic hyperreflexia include premature atrial and ventricular contractions, ${ }^{1,8}$ sinus tachycardia and bradycardia, ${ }^{1}$ alterations in T-wave and U-wave amplitudes, ${ }^{1}$ atrial fibrillation, ${ }^{9,10}$ bigeminy $^{8}$ and conduction block. ${ }^{8}$ We are unaware of any reports of ventricular fibrillation in association with autonomic hyperreflexia.

In the present patient, symptoms of autonomic hyperreflexia preceded an initial event of ventricular fibrillation and cardiac arrest. During emergency department evaluation, wide variations in blood pressure were recorded and preceded ventricular fibrillation. These variations in blood pressure persisted throughout the initial hospitalization but subsequently returned to his baseline blood pressure. No other recognized etiology for the recurrent ventricular fibrillation was determined (e.g. hypoxic, metabolic, toxic, etc.), and the patient subsequently elected to have an implanted cardiac defibrillator placed. Electrophysiological testing failed to induce a ventricular arrhythmia; this testing was performed after much of the variability in blood pressure recordings resolved.
We hypothesize that heightened sympathetic adrenergic activity that occurred during an episode of autonomic hyperreflexia resulted in a cardiac arrhythmia leading to ventricular fibrillation and cardiac arrest. The witnessed recurrent ventricular fibrillation with successful cardiac resuscitation allowed a rare opportunity to observe this commonly fatal arrhythmia in the presence of autonomic hyperreflexia. In an unwitnessed setting, death would certainly have occurred.

It is interesting to speculate as to what extent autonomic hyperreflexia accounts for the deaths in persons with chronic spinal cord injuries at risk for this condition. Cardiac disease now accounts for a significant number of deaths in the spinal cord injury population. $^{12}$ Deaths from cardiac causes in this population occur at a younger age than has been seen in the general population. ${ }^{12}$ It is possible that as individuals with chronic spinal cord injury age, they become more susceptible to the cardiac effects of heightened sympathetic adrenergic activity occurring during episodes of autonomic hyperreflexia. This could account for some of the unexpected deaths attributable to cardiac disease.

The current case of autonomic hyperreflexia in a patient with C6 tetraplegia with recurrent ventricular fibrillation and cardiac arrest illustrates the profound effects of massive paroxysmal sympathetic activity associated with this condition.

\section{References}

1 Guttmann L, Whitteridge D. Effects of bladder distension on autonomic mechanisms after spinal cord injuries. Brain 1947; 70: $361-404$.

2 Kewalramani LS. Autonomic dysreflexia in traumatic myelopathy. Am J Phys Med 1980; 59: 1-21.

3 Kursh ED, Freehafer A, Persky L. Complications of autonomic dysreflexia. J Urol 1977; 118: 70 - 72.

4 Brown BT, Carrion HM, Politano VA. Guanethidine sulfate in the prevention of autonomic hyperreflexia. J Urol 1979; 122: 55 57

5 Head H, Riddoch G. The automatic bladder, excessive sweating and some other reflex conditions, in gross injuries of the spinal cord. Brain 1917; 40: 188-263.

6 Lindan R, Joiner B, Freehafer AA, Hazel C. Incidence and clinical features of autonomic dysreflexia in patients with spinal cord injury. Paraplegia 1980; 18: 285-292.

7 Wurster RD, Randall WC. Cardiovascular responses to bladder distension in patients with spinal transection. Am J Physiol 1975; 228: $1288-1292$

8 Guttmann L, Frankel HL, Paeslack V. Cardiac irregularities during labor in paraplegic women. Paraplegia 1965; 3: $141-151$.

9 Forrest GP. Atrial fibrillation associated with autonomic dysreflexia in patients with tetraplegia. Arch Phys Med Rehabil 1991; 72: $592-594$

10 Wayne EM, Vukov JG. Eye findings in autonomic hyperreflexia. Ann Ophthalmol 1977; 9: 41 - 42

11 Kiker JD, Woodside JR, Jelinek GE. Neurogenic pulmonary edema associated with autonomic dysreflexia. J Urol 1982; 128: $1038-1039$.

12 Whiteneck GG et al. Mortality, morbidity, and psychosocial outcomes of persons spinal cord injured more than 20 years ago. Paraplegia 1992; 30: 617-630. 